\title{
Effect of food on the pharmacokinetic characteristics of a single oral dose of LCBOI-037I, a novel oxazolidinone antibiotic
}

This article was published in the following Dove Press journal:

Drug Design, Development and Therapy

\author{
Jung Sunwoo' \\ Yu Kyong Kim' \\ Yewon Choi' \\ Kyung-Sang Yu' \\ Heesook $\mathrm{Nam}^{2}$ \\ Young Lag Cho ${ }^{2}$ \\ Seonghae Yoon ${ }^{3}$ \\ Jae-Yong Chung ${ }^{3}$
}

'Department of Clinical Pharmacology and Therapeutics, Seoul National

University College of Medicine and Hospital, Seoul, ${ }^{2}$ LegoChem Biosciences, Inc., Daejeon,

${ }^{3}$ Department of Clinical Pharmacology and Therapeutics, Seoul National University College of Medicine and Bundang Hospital, Seongnam, Republic of Korea
Correspondence: Jae-Yong Chung Department of Clinical Pharmacology and Therapeutics, Seoul National University College of Medicine and Bundang Hospital, 82, Gumi-ro, 173 Beon-gil, Bundang-gu, Seongnam-si, Gyeonggi-do, 13620, Republic of Korea

$\mathrm{Tel}+82317873955$

Fax +82317874045

Email jychung@snubh.org
Background: LCB01-0371 is a novel oxazolidinone antibiotic that blocks protein production by binding to bacterial $23 \mathrm{~S}$ ribosomes. This antibiotic is active against Gram-positive bacteria. This study aimed to evaluate the effect of food on the pharmacokinetics (PKs) of LCB01-0371 and evaluate its safety profile.

Subjects and methods: A randomized, open-label, two-way crossover study was performed in 18 healthy Korean male subjects. All subjects received a single oral $800 \mathrm{mg}$ dose of LCB01-0371 in each period under fed or fasting condition with a 7-day washout in between. The fed condition was defined as consumption of a meal of $800-1,000 \mathrm{kcal}$ containing $~ 50 \%$ of fat content. Serial blood samples were collected over $24 \mathrm{~h}$ after dosing, and the PK parameters were calculated by noncompartment analysis. All available data of the subjects who received LCB01-0371 at least once were included in the safety data summaries.

Results: In the fed condition, both the maximum plasma concentration $\left(C_{\max }\right)$ and the total systemic exposure (area under the plasma concentration-time curve from time zero to the last observed time point $\left[\mathrm{AUC}_{\text {last }}\right.$ ]) decreased by $\sim 33 \%$ and $10 \%$, respectively. The time to reach $C_{\max }$ was delayed by $\sim 1.25 \mathrm{~h}$ in the fed condition, whereas the mean elimination half-life remained similar in both conditions. In the fed/fasting condition, the geometric mean ratios and $90 \% \mathrm{CI}$ of the $C_{\max }$ and $\mathrm{AUC}_{\text {last }}$ were $0.666(0.470-0.945)$ and 0.897 (0.761-1.057), respectively. There were no drug-related adverse events (AEs) or serious AEs.

Conclusion: Although the $T_{\max }$ after a single oral $800 \mathrm{mg}$ dose of LCB01-0371 was slightly delayed under the fed condition compared to the fasting condition, the total systemic exposure was similar under both conditions. Therefore, LCB01-0371 could be administered regardless of food intake.

Keywords: antibiotic resistance, gram-positive bacteria, clinical trial

\section{Introduction}

Over the course of the 21 st century, bacterial antibiotic resistance has become a global public health issue. ${ }^{1}$ Due to the increasing number of bacteria that are resistant to available conventional antibiotics, there is a need for the development of antibiotics with new mechanisms of action for treating diseases caused by multidrug-resistant bacteria.

The oxazolidinone antibiotics are a relatively new class of drugs developed in response to the increasing antibiotic resistance. This class has strong antimicrobial activity against most Gram-positive bacteria and organisms resistant to other agents, and they work by binding to the $\mathrm{V}$ domain of $23 \mathrm{~S}$ rRNA to block the initiation of complex formation during protein synthesis. ${ }^{2}$ Linezolid and tedizolid, antibiotics of the 
same family with a similar mechanism of action, have already received approval and are currently being marketed.

LCB01-0371 is a new oxazolidinone with a cyclic amidrazone developed by LegoChem Biosciences Inc. (Daejeon, Republic of Korea). ${ }^{2,3}$ A randomized, double-blind, placebocontrolled, multiple-dose ascending study of LCB01-0371 was previously conducted. ${ }^{4}$ For 21 days, the first treatment group received $800 \mathrm{mg}$ once a day, the second group received $800 \mathrm{mg}$ twice a day, and the third group received 1,200 mg twice a day. This study closely monitored the percentage change from baseline of absolute neutrophil count and red blood cell count. It was determined that long-term administration of LCB01-0371 was well tolerated.

The effect of food on the pharmacokinetics (PKs) of oxazolidinone drugs has been investigated in other studies. A slight decrease in $C_{\max }$ and a delay in $T_{\max }$ were observed after the administration of a single $375 \mathrm{mg}$ dose of linezolid. ${ }^{5}$ However, the oral bioavailability of linezolid was not affected by food, indicating that linezolid can be taken with or without food. ${ }^{6}$ After administration of a single $600 \mathrm{mg}$ dose of tedizolid phosphate, $C_{\max }$ was reduced to $27.3 \%$ and $T_{\max }$ was delayed under fed conditions compared with fasting conditions. Gastric emptying time was delayed in association with solid meals because tedizolid phosphate has low predicted membrane permeability, and the gastric juice within the stomach does not contain phosphatase enzymes. The geometric mean ratio (GMR) of the area under the plasma concentration-time curve (AUC) was similar between fed and fasting conditions. ${ }^{7}$ Because the AUCs of linezolid and tedizolid were similar under fed and fasting conditions, it is possible to administer them irrespective of diet.

In a similar context to the above study, the present study was performed to evaluate the effect of food on the PK characteristics, safety, and tolerability of a single oral $800 \mathrm{mg}$ dose of LCB01-0371.

\section{Subjects and methods Subjects and treatments}

This study was approved by the Institutional Review Board of Seoul National University Bundang Hospital (no B-1507-307-009) and registered in the clinical trial registry (ClinicalTrials.gov, no NCT02538003). Moreover, this study was conducted on the basis of the ethical principles of the Declaration of Helsinki ${ }^{8}$ and the rules of Korean Good Clinical Practices. ${ }^{9}$

Healthy Korean male adults aged between 20 and 40 years and with a body mass index (BMI) of $19-28 \mathrm{~kg} / \mathrm{m}^{2}$ were enrolled for the study. Written informed consent was obtained from all subjects before the screening procedure.
To be enrolled, aspartate aminotransferase, alanine aminotransferase, gamma-glutamyl transferase, and total bilirubin levels were required to be below the upper limit of normal. Subjects with clinically significant disease history or with an inability to digest high-fat, high-calorie diets (800-1,000 kcal, $\sim 50 \%$ of fat content) were excluded. Those who were scheduled to use selective serotonin reuptake inhibitor, monoamine A and B inhibitors, or drugs with serotonin activity for 2 weeks prior to randomization were also excluded. Those who had hypersensitivities to oxazolidinone (eg, linezolid and tedizolid) or other drugs (eg, aspirin and antibiotics) were excluded; however, subjects with inactive allergic rhinitis were eligible for participation. Furthermore, subjects who were found to be unsuitable for clinical trials at the discretion of the investigators were also restricted from participating in this study.

This study had a randomized, open-label, single-dose, two-by-two crossover design. A total of 18 subjects were randomized, and 9 subjects were enrolled in each group. Subjects in group 1 were administered a single $800 \mathrm{mg}$ dose of LCB01-0371 with $240 \mathrm{~mL}$ of water in the fasting condition at period 1 and in the fed condition at period 2 . Subjects in group 2 were administered a single $800 \mathrm{mg}$ dose of LCB01-0371 with $240 \mathrm{~mL}$ of water in the fed condition in period 1 and in the fasting condition in period 2 . The fasting condition meant that at least $10 \mathrm{~h}$ of fasting was maintained prior to the administration of the drug. According to the US Food and Drug Administration guidance on food effect studies, ${ }^{10}$ subjects who were scheduled for postprandial administration consumed a high-fat diet (800-1,000 kcal containing $\sim 50 \%$ of fat) within $20 \mathrm{~min}$. The subjects took $800 \mathrm{mg}$ LCB01-0371 $\geq 30$ min after the meal start time. The washout period for each group was 7 days.

\section{Blood sample collection}

Blood was collected for PK evaluation 15 times for each period up to $24 \mathrm{~h}$ after the drug administration, at 0 (predose) and $0.25,0.5,0.75,1,1.5,2,2.5,3,4,6,8,10,12$, and $24 \mathrm{~h}$ postdose. A volume of $6 \mathrm{~mL}$ of blood was collected in heparinized tubes and centrifuged at $1,800 \mathrm{rpm}$ at $4^{\circ} \mathrm{C}$ for $8 \mathrm{~min}$, from which three aliquots of $0.8 \mathrm{~mL}$ of plasma were transferred to Eppendorf tubes and maintained at $-70^{\circ} \mathrm{C}$ until the concentration analysis.

\section{Determination of plasma drug concentration}

The plasma concentration of LCB01-0371 was determined using a validated liquid chromatography (LC) coupled with a mass spectrometry (MS) method. 
LC was performed using a nanospace SI-2 high-performance liquid chromatography (HPLC) system (Shiseido, Tokyo, Japan) consisting of a binary pump, a vacuum degasser, an autosampler, and a column oven compartment. The analytes were separated on an Atiantis dC18 column $(100 \times 2.1 \mathrm{~mm}$, $3 \mu \mathrm{m}$ ) (Waters, Milford, MA, USA) set at $40^{\circ} \mathrm{C}$. The mobile phase consisting of $1 \mathrm{mM}$ ammonium formate and acetonitrile $(80: 20, \mathrm{v} / \mathrm{v})$ was used in an isocratic condition at a flow rate of $0.3 \mathrm{~mL} / \mathrm{min}$.

MS was performed using a Quantum Ultra Triple Quadrupole Mass Spectrometer (Thermo Fisher Scientific, Waltham, MA, USA). Xcalibur software (Version 2.1.0) was used for system control and data processing. Electrospray ionization was operated in the positive ionization mode to detect LCB01-0371. The ion spray voltage was set at $4,000 \mathrm{~V}$, and the capillary temperature was set at $300^{\circ} \mathrm{C}$. A multiple reaction monitoring scan mode was used in the mass spectrometer.

To simplify the extraction steps and optimize the chromatographic conditions, samples were prepared using protein precipitation from human plasma and target compounds were analyzed using the LC/MS/MS detection. We evaluated selectivity, sensitivity, linearity, accuracy and precision, recovery, carryover, matrix effect, batch size, dilution factor, short-term temperature stability (at room temperature), postpreparative stability, freeze-thaw stability, long-term stability $\left(<-70^{\circ} \mathrm{C}\right)$, stock solution stability, and working solution stability.

After validation, no endogenous interference peak was observed at the same retention time as LCB01-0371 and the signal-to-noise ratio at the lower limit of quantification (LLOQ) concentration was $>5 \mathrm{ng} / \mathrm{mL}$. The concentration range in plasma was $5-20,000 \mathrm{ng} / \mathrm{mL}$, and the linearity of calibration curve was satisfied with the criteria of determination coefficient $\left(r^{2}\right) \geq 0.9900$. We determined accuracy and precision at four concentrations (LLOQ, low, medium, and high) during inter- and intra-batch. The accuracy was 85\%-115\% (LLOQ: $80 \%-120 \%$ ), and the precision was within 15\% (LLOQ: within 20\%). The stability results of analyte were in an acceptable change of $\pm 15 \%$. In addition, the change $(\%)$ between the reference sample and the test sample was confirmed to be within $\pm 10 \%$ for stock solution and working solution stability.

For sample preparation, to a polypropylene tube containing $95 \mu \mathrm{L}$ of blank plasma, $5 \mu \mathrm{L}$ of LCB01-0371 working solution and $10 \mu \mathrm{L}$ of internal standard (IS) were added and vortexed briefly. Thereafter, $1 \mathrm{~mL}$ of acetonitrile was added to mixture to precipitate the protein. The mixture was vortexed for $5 \mathrm{~min}$ and centrifuged at 13,000 rpm for $5 \mathrm{~min}$. Deionized water $(1 \mathrm{~mL})$ was added to the $100 \mu \mathrm{L}$ upper layer.
The mixtures were collected in a vial, and $5 \mu \mathrm{L}$ of a sample was injected into the HPLC-MS/MS system for analysis.

\section{PK analysis}

PK analysis was performed for those who completed all blood collection schedules. The actual blood sampling time points were used for PK analysis for each subject.

From the obtained data, the following PK parameters were calculated with the noncompartmental method using the Phoenix WinNonlin software, Version 6.4 (Certara, Princeton, NJ, USA). The maximum plasma concentration $\left(C_{\max }\right)$ and the time to reach $C_{\text {max }}\left(T_{\max }\right)$ were obtained directly from the observed values. If the plasma concentration was below the LLOQ before $T_{\max }$, the plasma concentration was considered zero. If the plasma concentration observed after $T_{\max }$ was below the LLOQ, the plasma concentration was considered missing. The AUC from time zero to the last observed time point $\left(\mathrm{AUC}_{\text {last }}\right)$ was determined using the linear trapezoidal method for ascending concentrations and the log trapezoidal method for descending concentrations. Extrapolation beyond the last plasma concentration was performed to determine the AUC from time zero to infinity $\left(\mathrm{AUC}_{\mathrm{inf}}\right)$. The elimination half-life $\left(t_{1 / 2}\right)$ was calculated as the natural logarithm of two divided by $\lambda_{z}$, where $\lambda_{z}$ was the terminal elimination rate constant estimated from a regression line of log-transformed plasma concentrations versus time over the terminal log-linear portion.

\section{Safety and tolerability assessments}

All subjects who received at least one dose were evaluated for safety. Assessment criteria included adverse event (AE) monitoring, physical examination, vital signs, 12-lead electrocardiogram, and clinical laboratory tests. Subjects were instructed to report to the investigator or medical personnel if there were any AEs throughout the entire study. All AEs reported by the subjects were recorded, and the investigators assessed the relationship with the investigational product.

\section{Statistical analyses}

Demographic characteristics were obtained using descriptive statistics (mean, SD). All PK parameters are displayed as mean $\pm \mathrm{SD}$ and coefficient of variation (\%), except for $T_{\max }$, which was represented as median and range.

The GMRs were calculated from the analysis of variance model, including sequence, period, and fed/fasting condition as fixed effects and the subject nested within sequence as a random effect. All statistical analyses were performed 
using the SAS software (Version 9.4; SAS Institute Inc., Cary, NC, USA).

\section{Results}

\section{Demographics}

The purpose of this study was to compare the PK characteristics of a single oral administration of LCB01-0371 under fed and fasting conditions. For the food effect study, the number of subjects was set to $\sim 20$ or more, with the minimum number of subjects being at least 12 . The study was conducted with nine healthy subjects, which was slightly more than the minimum number of subjects to determine the effect of food on the PKs of the investigational product.

Nineteen healthy Korean male volunteers were enrolled and randomized. One subject withdrew consent before the dosing at period 1, and another subject withdrew consent before starting period 2. A total of 17 subjects completed the study, and the demographic characteristics are shown in Table 1.

The mean \pm SD of age, height, weight, and BMI of all 19 randomly enrolled subjects were $25.1 \pm 3.3$ years, $171.3 \pm 7.3 \mathrm{~cm}, 69.8 \pm 8.2 \mathrm{~kg}$, and $23.8 \pm 2.6 \mathrm{~kg} / \mathrm{m}^{2}$, respectively. The demographic characteristics were similar in both groups.

\section{PK}

PK analysis was performed in 17 subjects. A summary of the PK parameters after a single administration of LCB01$0371800 \mathrm{mg}$ under fed and fasting conditions and, also, the GMR and $90 \% \mathrm{CI}$ of $C_{\max }, \mathrm{AUC}_{\text {last }}$, and $\mathrm{AUC}_{\text {inf }}$ for fed and fasting conditions are shown in Table 2. The mean plasma concentrations of LCB0-0371 versus time profiles under each condition are shown in Figure 1. Individual changes in PK parameters after a single $800 \mathrm{mg}$ dose of LCB01-0371 under each condition are shown in Figure 2.

Table I Demographic characteristics of subjects according to groups

\begin{tabular}{llll}
\hline $\begin{array}{l}\text { Demographic } \\
\text { data }\end{array}$ & \multicolumn{4}{l}{\begin{tabular}{l} 
LCB0I-037 I 800 $\mathbf{~ m g}^{\mathrm{a}}$ \\
\cline { 2 - 4 }
\end{tabular}} & $\begin{array}{l}\text { Group I } \\
\text { (fasted-fed } \\
\text { sequence) }\end{array}$ & $\begin{array}{l}\text { Group 2 } \\
\text { (fed-fasted } \\
\text { sequence) }\end{array}$ & $\begin{array}{l}\text { Total } \\
(\mathbf{N}=19)\end{array}$ \\
\hline Age (years) & $26.0 \pm 3.0$ & $24.3 \pm 3.4$ & $25.1 \pm 3.3$ \\
Height $(\mathrm{cm})$ & $170.2 \pm 7.3$ & $172.4 \pm 7.4$ & $171.3 \pm 7.3$ \\
Weight $(\mathrm{kg})$ & $69.9 \pm 9.4$ & $69.6 \pm 7.5$ & $69.8 \pm 8.2$ \\
BMI $\left(\mathrm{kg} / \mathrm{m}^{2}\right)$ & $24.2 \pm 3.0$ & $23.4 \pm 2.2$ & $23.8 \pm 2.6$ \\
\hline
\end{tabular}

Notes: a Data presented as mean \pm SD. ${ }^{b} \mathrm{LCBOI}-037 \mathrm{I}$ administered under fasting conditions followed by fed conditions. 'LCBOI-037I administered under fed conditions followed by fasting conditions.

Abbreviation: BMI, body mass index.
The median $T_{\max }$ of LCB01-0371 under fed conditions was delayed from 0.75 to $2 \mathrm{~h}$ compared to the median $T_{\max }$ of LCB01-0371 under fasting conditions ( $P$-value $<0.0001)$, whereas the $t_{1 / 2}$ was not statistically significantly different between the two groups. Under fed conditions, $C_{\max }$ significantly decreased by $\sim 33.4 \%$ from that in fasting conditions. However, $\mathrm{AUC}_{\text {last }}$ and $\mathrm{AUC}_{\text {inf }}$ were similar between fed and fasting conditions. GMRs $(90 \% \mathrm{CI})$ of $C_{\max }$ and $\mathrm{AUC}_{\text {last }}$ were 0.666 (0.470-0.945) and 0.897 (0.761-1.057), respectively.

\section{Safety and tolerability}

The safety and tolerability assessment was performed in 18 subjects.

A total of seven mild AEs in two subjects were reported after an oral single administration of LCB01-0371 under fed or fasting conditions. Four AEs (rhinorrhea, sore throat, headache, and noncardiac chest pain) were reported in two subjects under the fasting condition, and three AEs (cough, fever, and noncardiac chest pain) were reported in two subjects under the fed condition. There were no clinically significant differences between before and after dosing in vital signs, physical examinations, 12-lead electrocardiogram, and clinical laboratory tests.

\section{Discussion}

This study was the first to evaluate the effect of food on the PKs of LCB01-0371. Because there were no statistically significant differences in AUC between the fed and fasting conditions, we concluded that food did not significantly influence the PKs of LCB01-0371.

AUC divided by minimum inhibitory concentration (AUC/MIC) is an indicator used to estimate the efficacy of antibiotics according to concentration-time characteristics, ${ }^{11}$ especially for antibiotics that have a long postantibiotic effect (PAE). ${ }^{12}$ For the PK/pharmacodynamics (PD) antibacterial assay for LCB01-0371, a mouse model of bacteria-induced infection in thighs was used. The AUC/MIC parameter was well correlated with efficacy in this experiment. In addition, when the PK/PD study of LCB01-0371 was performed using a neutropenic mouse thigh infection model, the PAE of LCB01-0371 was $16 \mathrm{~h}$ at a dose of $40 \mathrm{mg} / \mathrm{kg}$. This PAE is very long compared to the PAE of linezolid, which is $\sim 1-3$ h. ${ }^{11,13}$ Because there were no significant differences in $\mathrm{AUC}_{\text {last }}$ between the fed and fasting conditions in this study, the effect of food on the efficacy of LCB01-0371 is expected to be negligible for bacteria strains with a common MIC causing clinical infection. 
Table 2 Summary of LCBOI-037I pharmacokinetic parameters after a single $800 \mathrm{mg}$ dose of LCBOI-037I under fed and fasting conditions

\begin{tabular}{|c|c|c|c|c|c|}
\hline \multirow{3}{*}{$\begin{array}{l}\text { Pharmacokinetic } \\
\text { parameter }\end{array}$} & \multicolumn{4}{|c|}{ LCBOI-037I $800 \mathrm{mg}$} & \multirow[t]{3}{*}{$\mathrm{GMR}^{\mathrm{b}}(90 \% \mathrm{Cl})$} \\
\hline & \multicolumn{2}{|l|}{ Fed $(\mathbf{N}=\mid 7)$} & \multicolumn{2}{|l|}{ Fasted $(\mathbf{N}=17)$} & \\
\hline & Mean $\pm \mathbf{S D}^{\mathbf{a}}$ & CV (\%) & Mean $\pm \mathbf{S D}^{\mathbf{a}}$ & CV (\%) & \\
\hline$T_{\max }(\mathrm{h})$ & $2.00(1.00-6.00)$ & & $0.75(0.50-3.00)$ & & - \\
\hline$C_{\max }(\mathrm{mg} / \mathrm{L})$ & $7.26 \pm 2.58$ & 35.6 & $11.74 \pm 5.38$ & 45.9 & $0.666(0.470-0.945)$ \\
\hline $\mathrm{AUC}_{\text {last }}(\mathrm{mg} \mathrm{h} / \mathrm{L})$ & $|8.25 \pm 4.5|$ & 24.7 & $20.46 \pm 5.23$ & 25.6 & $0.897(0.76 \mathrm{I}-\mathrm{I} .057)$ \\
\hline $\mathrm{AUC}_{\text {inf }}(\mathrm{mg} \mathrm{h} / \mathrm{L})$ & $|8.4| \pm 4.5 \mid$ & 24.5 & $20.56 \pm 5.24$ & 25.5 & $0.900(0.765-1.060)$ \\
\hline$t_{1 / 2}(\mathrm{~h})$ & $1.53 \pm 0.14$ & 9.0 & $1.68 \pm 0.44$ & 26.0 & - \\
\hline
\end{tabular}

Notes: $A \cup C_{\text {last }}$, AUC from time zero to the last observed time point; $A \cup C_{\text {inf }}$, AUC from time zero to infinity; $C_{\text {max }}$, maximum plasma concentration; $t_{1 / 2}$, terminal elimination half-life; $T_{\max }$, time to reach maximum plasma concentration. ${ }^{a}$ Data are presented as mean \pm SD except for $T_{\max }$, for which median (minimum-maximum) is presented. 'GMR of pharmacokinetic parameter under fed condition to fasting condition. "-" = not applicable.

Abbreviations: AUC, area under the plasma concentration-time curve; CV, coefficient of variation; GMR, geometric mean ratio.

According to linezolid surveillance results for the USA in 2014 (LEADER Surveillance Program),${ }^{14}$ minimum inhibitory concentration to inhibit the growth of $50 \%$ of organisms $\left(\mathrm{MIC}_{50}\right)$ and minimum inhibitory concentration to inhibit the growth of $90 \%$ of organisms $\left(\mathrm{MIC}_{90}\right)$ values against Staphylococcus aureus (47.2\% were methicillin-resistant $S$. aureus [MRSA]) for linezolid were both $1 \mathrm{mg} / \mathrm{L}$. In the study in China, $\mathrm{MIC}_{50}$ and $\mathrm{MIC}_{90}$ against MRSA for tedizolid were both $0.25 \mathrm{mg} / \mathrm{L}$, whereas these two values were $2 \mathrm{mg} / \mathrm{L}$ for linezolid. $\mathrm{MIC}_{50}$ and $\mathrm{MIC}_{90}$ values against methicillin-sensitive $S$. aureus (MSSA) for tedizolid were 0.25 and $0.5 \mathrm{mg} / \mathrm{L}$, respectively, whereas both values were $2 \mathrm{mg} / \mathrm{L}$ for linezolid. ${ }^{15}$ The static dose PD targets for linezolid and tedizolid were almost identical, with AUC/MIC ratios 19 and 20, respectively. ${ }^{16}$ Based on these results, the effective AUC of LCB01-0371 was calculated to be 5-10 $\mathrm{mg} \mathrm{h} / \mathrm{L}$, assuming a target AUC/MIC of 20 for LCB01-0371. The nonclinical data for LCB01-0371 indicated a similar MIC to linezolid for MRSA and MSSA, which were $2 \mathrm{mg} / \mathrm{L}$ for both. Consequently, the target AUC that satisfied the static effect was $5-10 \mathrm{mg} \mathrm{h} / \mathrm{L}$. All subjects reached an AUC that could satisfy the bacteriostatic effect after the oral administration of $800 \mathrm{mg}$ of LCB01-0371. Therefore, the use of $800 \mathrm{mg}$ of LCB01-0371 is considered appropriate. Multiple doses of $800 \mathrm{mg}$ of LCB01-0371 showed good tolerability and safety, ${ }^{17}$ and when combined with the above results, it is expected that the $800 \mathrm{mg}$ dose of LCB01-0371 will show the desired efficacy and safety in patients.

Under fed conditions, $C_{\max }$ decreased by $26.3 \%$ after the administration of tedizolid and AUC was similar to that under the fasting condition. ${ }^{7}$ This is comparable to the results of this study, where the $C_{\max }$ decreased and the AUC was

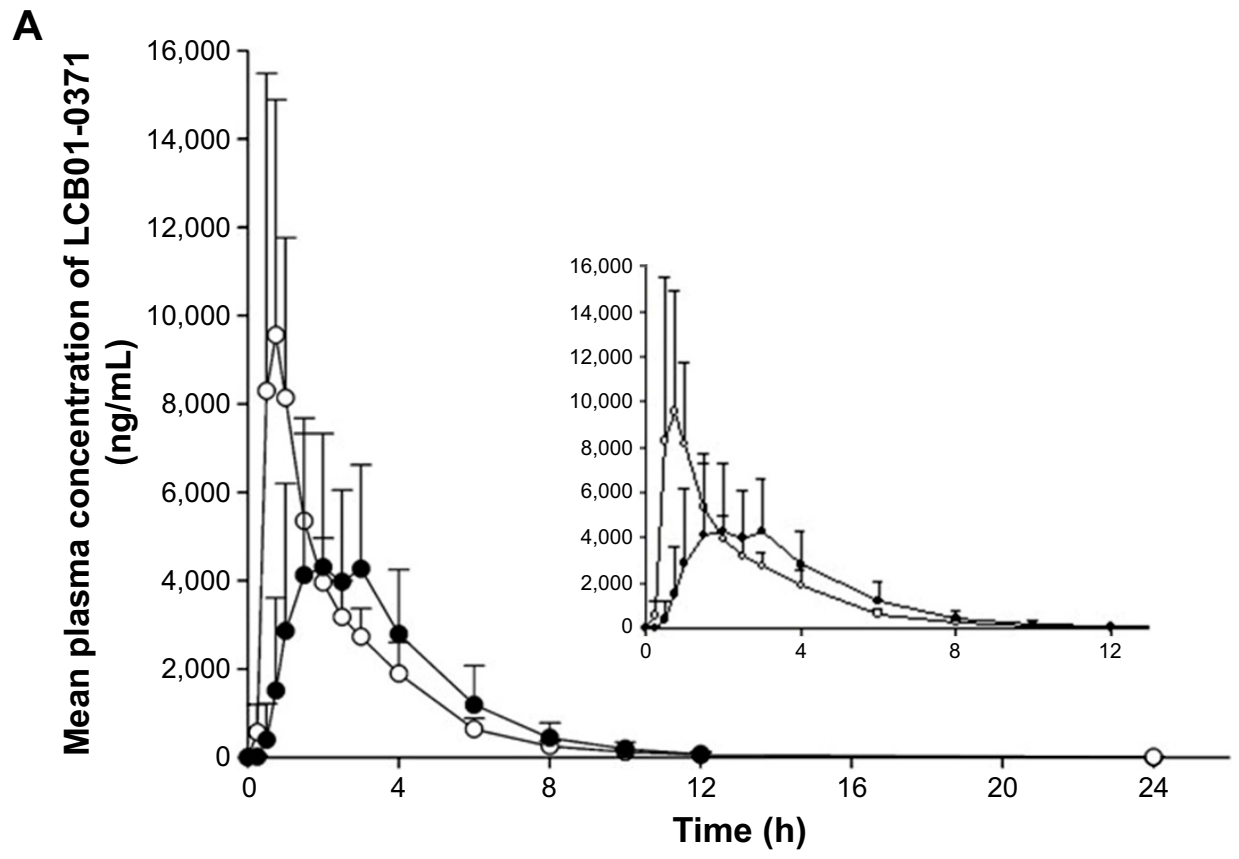

Figure I (Continued) 


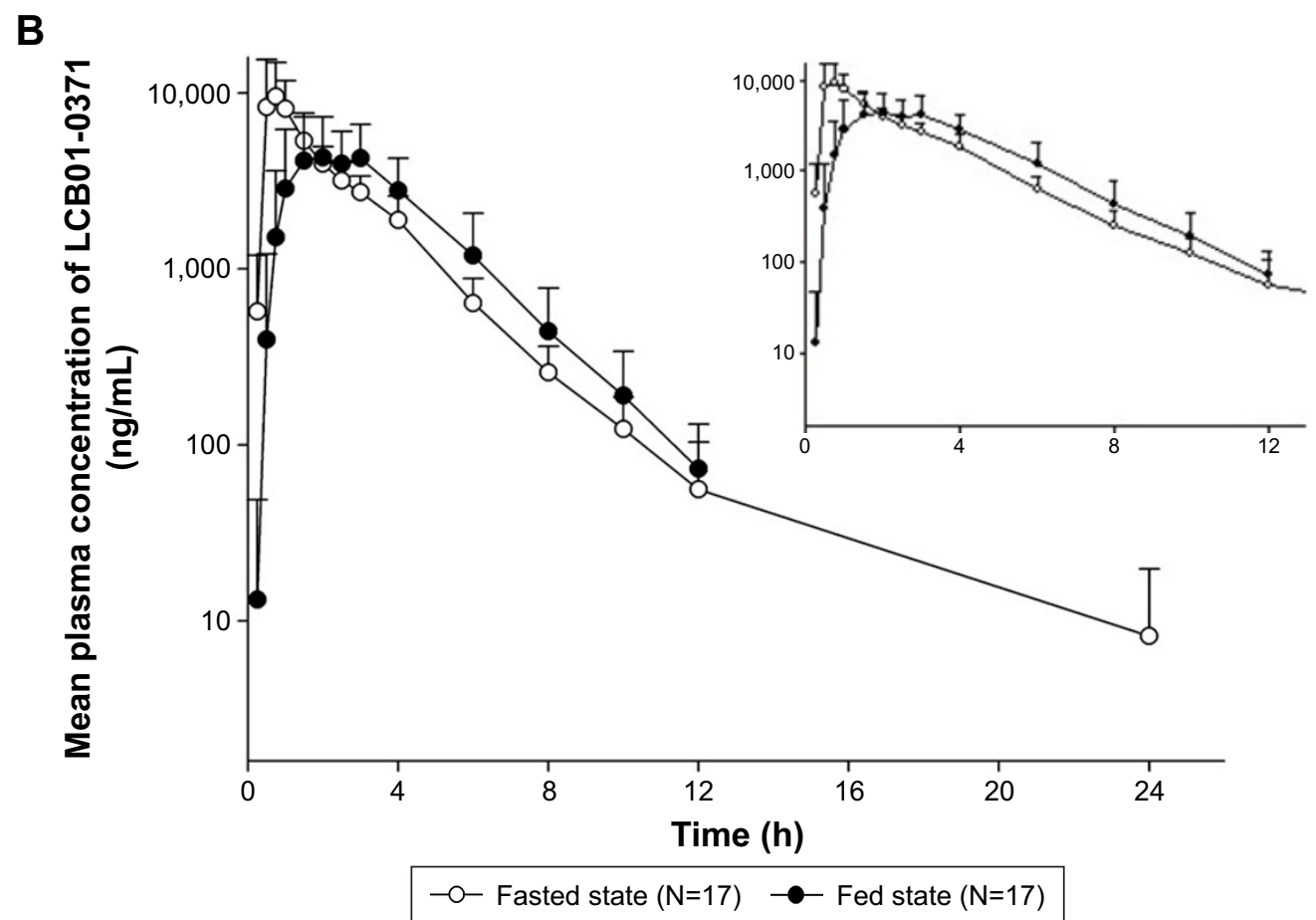

Figure I Mean plasma LCBOI-037I concentration-time profiles after a single $800 \mathrm{mg}$ dose of LCB0I-037I under fed and fasting conditions.

Note: Data are presented as mean+SD (A: linear scale and B: log-linear scale; small graph in each panel represents the time of 0-12 h in linear and log-linear scales, respectively).
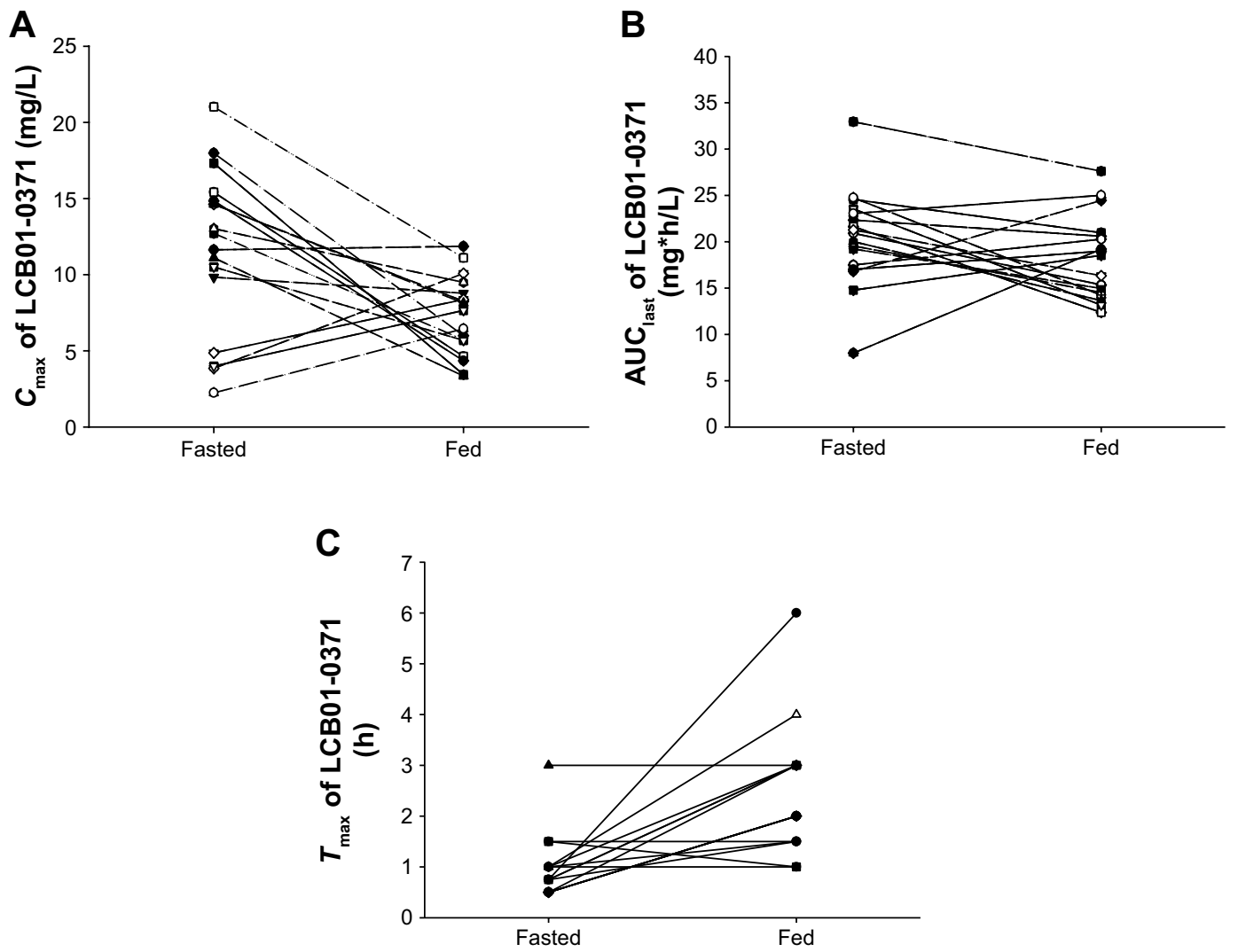

Figure 2 Individual changes in pharmacokinetic parameters after a single $800 \mathrm{mg}$ dose of LCBOI-037I under fed and fasting conditions.

Notes: (A) The changes in $C_{\max }$ under fasting and fed conditions. (B) The changes in $A \cup C_{\text {last }}$ and (C) the changes in median $T_{\max }$ under fasting and fed conditions. $C_{\max }$, maximum plasma concentration; $A \cup C_{\text {last }}, A \cup C$ from time zero to the last observed time point; $T_{\max }$, time to reach $C_{\max }$

Abbreviations: AUC, area under the plasma concentration-time curve; $C_{\max }$, maximum plasma concentration; $A \cup C_{\text {last }}, A U C$ from time zero to the last observed time point; $T_{\max }$, time to reach $C_{\max }$. 
similar under fed and fasting conditions. However, the $T_{\max }$ and $t_{1 / 2}$ of LCB01-0371 and tedizolid were different. Under fasting conditions, the $T_{\max }$ of LCB01-0371 ranged from 0.50 to $3.00 \mathrm{~h}$, whereas that of tedizolid ranged from 1.5 to $3.0 \mathrm{~h}$. Under fed conditions, the $T_{\text {max }}$ of LCB01-0371 ranged from 1.00 to $6.00 \mathrm{~h}$, whereas that of tedizolid ranged from 4.0 to $12.0 \mathrm{~h}$. The $t_{1 / 2}$ of LCB01-0371 was shorter than that of tedizolid (LCB01-0371: 1.53-1.68 h, tedizolid: 10.4-10.9 h).

Linezolid is available as in both oral and intravenous formulations, and the bioavailability of the oral tablet is $\sim 100 \%$, meaning that the oral and intravenous doses are the same. The $T_{\max }$ of oral linezolid was slightly delayed under fed conditions (1.0-1.7 to $1.5-2.0 \mathrm{~h}){ }^{6}$ The mean $C_{\max }$ was $23 \%$ higher in fasting subjects than in high-fat meal recipients. Because the AUC was similar under fed and fasting conditions, the overall extent of absorption was the same. ${ }^{5}$ This is also comparable with the results obtained in the present study.

LCB01-0371 did not easily dissolve in water, but it was soluble in $50 \%$ acetonitrile mixed with water. In addition, the permeability of LCB01-0371 was medium grade when measured using the parallel artificial membrane permeability assay (PAMPA), which was developed to determine the degree of permeation across the gastrointestinal tract. ${ }^{18}$ The $\mathrm{p} K_{\mathrm{a}}$ of LCB01-0371 was $\sim 4.82$, indicating that it is a weak acid. Considering these physiochemical characteristics of LCB01-0371, the ionization of LCB01-0371 would be expected to increase when gastric $\mathrm{pH}$ increases. A high-fat meal increases gastric $\mathrm{pH}$ and gastric emptying time. Ionization of the chemical leads to a lower rate of absorption into the gastrointestinal tract. Therefore, $C_{\max }$ should decrease in fed conditions. AUC under fed and fasting conditions may have been similar, because LCB01-0371 has an amphiphilic nature that results in increased absorption in the small intestine even if the gastric emptying time is delayed.

There were some limitations to this study. Although multiple dosing design studies are not necessary to determine the effect of food on the PKs of a drug, multiple dosing of antibiotics is common in the clinical setting; therefore, further related studies are required. In addition, because LCB01-0371 is currently under development, the expected effective dose is still unknown. Therefore, the time above MIC, which is important for oxazolidinone efficacy, ${ }^{6}$ has yet to be determined. Further studies are required to determine the expected effective dose of LCB01-0371.

\section{Conclusion}

The total systemic exposure of $800 \mathrm{mg}$ of LCB01-0371 was similar under fed and fasting conditions. This indicated that the effectiveness of LCB01-0371 is based on AUC; it might be administered irrespective of diet. There was no significant difference in safety after a single dose of $800 \mathrm{mg}$ of LCB010371 under fed and fasting conditions.

\section{Acknowledgment}

This study was sponsored by LegoChem Biosciences Inc., Daejeon, Korea.

\section{Author contributions}

All the authors contributed significantly to the concept, design, implementation, analysis, and interpretation of the data. Each also played an important role in drafting, reviewing, and revising this article and gave final approval of the version to be published. Furthermore, all authors were responsible for properly investigating and resolving issues related to the accuracy and integrity of all parts of this article.

\section{Disclosure}

Heesook Nam and Young Lag Cho are LegoChem Biosciences, Inc. employees. The other authors report no conflicts of interest in this work.

\section{References}

1. Sharma VK, Johnson N, Cizmas L, McDonald TJ, Kim H. A review of the influence of treatment strategies on antibiotic resistant bacteria and antibiotic resistance genes. Chemosphere. 2016;150:702-714.

2. Jeong J-W, Jung S-J, Lee H-H, et al. In vitro and in vivo activities of LCB01-0371, a new oxazolidinone. Antimicrob Agents Chemother. 2010; 54(12):5359-5362.

3. Lee HH, Lee SR, Kwak JH. Resistance mechanism of enterococcus faecalis to LCB01-0371, a new oxazolidinone. Radiat Oncol J. 31(2). Available from: http://scholar.dkyobobook.co.kr/searchDetail. laf?barcode=4010023946472. Accessed May 11, 2018.

4. Choi Y, Lee SW, Kim A, et al. Safety, tolerability and pharmacokinetics of 21 day multiple oral administration of a new oxazolidinone antibiotic, LCB01-0371, in healthy male subjects. J Antimicrob Chemother. 2018;73(1):183-190.

5. Welshman IR, Sisson TA, Jungbluth GL, Stalker DJ, Hopkins NK. Linezolid absolute bioavailability and the effect of food on oral bioavailability. Biopharm Drug Dispos. 2001;22(3):91-97.

6. Stalker DJ, Jungbluth GL. Clinical pharmacokinetics of linezolid, a novel oxazolidinone antibacterial. Clin Pharmacokinet. 2003;42(13): 1129-1140.

7. Flanagan SD, Bien PA, Munoz KA, Minassian SL, Prokocimer PG. Pharmacokinetics of tedizolid following oral administration: single and multiple dose, effect of food, and comparison of two solid forms of the prodrug. Pharmacotherapy. 2014;34(3):240-250.

8. Association WM. Declaration of Helsinki: Ethical principles for medical research involving human subjects. 64th WMA General Assembly; Fortaleza, Brazil; 2016.

9. Group ICoHW. ICH harmonised tripartite guideline: guideline for good clinical practice E6 (R1). Paper presented at: International Conference on Harmonisation of Technical Requirements for Registration of Pharmaceuticals for Human Use; 1996. Available from: https://www.ich. org/fileadmin/Public_Web_Site/ICH_Products/Guidelines/Efficacy/ E6/E6_R1_Guideline.pdf. Accessed May 05, 2018.

10. U.S. Department of Health and Human Services. Guidance for Industry: Food-Effect Bioavailability and Fed Bioequivalence Studies. FDA, Center for Drug Evaluation and Research (CDER); 2002. Available from: https://www.fda.gov/downloads/drugs/guidancecomplianceregulatory information/guidances/ucm070241.pdf. Accessed May 05, 2018. 
11. Andes D, Van Ogtrop M, Peng J, Craig W. In vivo pharmacodynamics of a new oxazolidinone (linezolid). Antimicrob Agents Chemother. 2002;46(11):3484-3489.

12. Lee D-G, Shin H-H. Pharmacokinetics and pharmacodynamics of antibiotics: general concepts and recent advances. Infect Chemother. 2008;40(3):140-147.

13. Athamna A, Athamna M, Medlej B, Bast D, Rubinstein E. In vitro post-antibiotic effect of fluoroquinolones, macrolides, $\beta$-lactams, tetracyclines, vancomycin, clindamycin, linezolid, chloramphenicol, quinupristin/dalfopristin and rifampicin on Bacillus anthracis. $J$ Antimicrob Chemother. 2004;53(4):609-615.

14. Flamm RK, Mendes RE, Hogan PA, Streit JM, Ross JE, Jones RN. Linezolid surveillance results for the United States (LEADER surveillance program 2014). Antimicrob Agents Chemother. 2016;60(4): $2273-2280$
15. Chen $\mathrm{H}$, Yang $\mathrm{Q}$, Zhang R, et al. In vitro antimicrobial activity of the novel oxazolidinone tedizolid and comparator agents against Staphylococcus aureus and linezolid-resistant Gram-positive pathogens: a multicentre study in China. Int J Antimicrob Agents. 2014;44(3):276.

16. Lepak AJ, Marchillo K, Pichereau S, Craig WA, Andes DR. Comparative pharmacodynamics of the new oxazolidinone tedizolid phosphate and linezolid in a neutropenic murine Staphylococcus aureus pneumonia model. Antimicrob Agents Chemother. 2012;56(11):5916-5922.

17. Choi Y, Yoon J, Lee S, et al. Safety, tolerability and pharmacokinetics of multiple oral administration of Lcb01-0371, a new oxazolidinone antibiotics, in healthy male subjects. Clin Pharmacol Ther. 2016;99:S88-S89.

18. Avdeef A, Nielsen PE, Tsinman O. PAMPA - a drug absorption in vitro model: 11. Matching the in vivo unstirred water layer thickness by individual-well stirring in microtitre plates. Eur J Pharm Sci. 2004;22(5):365-374.

\section{Publish your work in this journal}

Drug Design, Development and Therapy is an international, peerreviewed open-access journal that spans the spectrum of drug design and development through to clinical applications. Clinical outcomes, patient safety, and programs for the development and effective, safe, and sustained use of medicines are the features of the journal, which has also been accepted for indexing on PubMed Central. The manuscript management system is completely online and includes a very quick and fair peer-review system, which is all easy to use. Visit http://www.dovepress.com/testimonials.php to read real quotes from published authors.

Submit your manuscript here: http://www.dovepress.com/drug-design-development-and-therapy-journal 